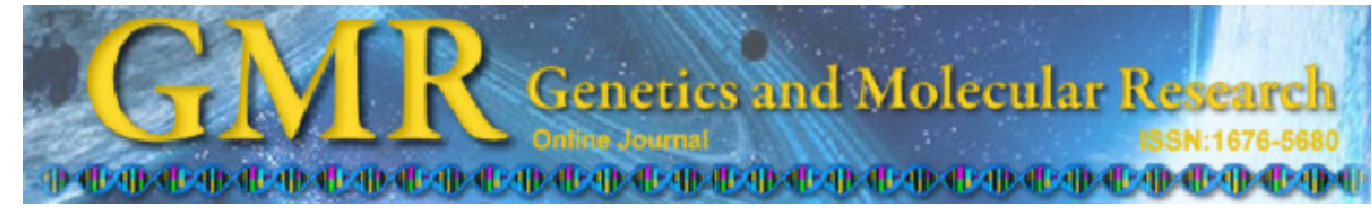

Case Report

\title{
Molecular characterization of microduplication 22q11.2 in a girl with hypernasal speech
}

\author{
Y. Soysal ${ }^{1}$, J. Vermeesch ${ }^{2}$, N.A. Davani ${ }^{2}$, N. Şensoy ${ }^{3}$, K. Hekimler ${ }^{1}$ \\ and $\mathrm{N}$. İmirzalığlu ${ }^{1}$ \\ ${ }^{1}$ Department of Medical Genetics, Faculty of Medicine, \\ Afyon Kocatepe University, Afyonkarahisar, Turkey \\ ${ }^{2}$ Center for Human Genetics, University Hospital, \\ Catholic University of Leuven, Leuven, Belgium \\ ${ }^{3}$ Department of Family Medicine, Faculty of Medicine, \\ Afyon Kocatepe University, Afyonkarahisar, Turkey \\ Corresponding author: Y. Soysal \\ E-mail: yasemin_soysal@yahoo.com
}

Genet. Mol. Res. 10 (3): 2148-2154 (2011)

Received February 21, 2011

Accepted August 16, 2011

Published September 21, 2011

DOI http://dx.doi.org/10.4238/vol10-3gmr1339

\begin{abstract}
We present a 12-year-old girl with karyotype 46,XX. A comparative genomic hybridization array revealed a $3.172-\mathrm{Mb}$ microduplication on $22 \mathrm{q} 11.2$. This chromosome $22 \mathrm{q} 11.2$ region microduplication has been described in patients with variable phenotypes; a large majority of them have identical 3-Mb duplications. The girl presented mild mental motor retardation, facial dysmorphism consisting of a long narrow face, widely spaced eyes, downslanting palpebral fissures, broad nasal base, short philtrum, thin upper lip, micro/retrognathia, low set and retroverted ears, microcephaly, higharched palate, hypoplastic teeth, and hypernasal speech. She had delayed psychomotor development and behavioral problems. Molecular characterization of patients differs greatly among reports and detailed molecular characterization and documentation are needed to better understand the effects of these duplications. This description of the phenotype of a patient with microduplication on $22 \mathrm{q} 11.2$ will contribute
\end{abstract}


to the growing knowledge regarding deletions and duplications of the 22q11.2 region; this is important to conclude whether $22 \mathrm{q} 11.2$ duplication is a microduplication syndrome or not.

Key words: Array-CGH; Hypernasal speech; Temporal hair loss; Microduplication 22q11.2; Mental retardation

\section{INTRODUCTION}

The chromosome 22q11.2 region is susceptible to well-known genomic disorders (Wentzel et al., 2008). DiGeorge/velocardiofacial syndrome (DGS/VCFS) (22q11.2 deletion syndrome, 22q11.2DS) is the most frequently identified genomic disorder of the region with a prevalence of $4 / 10,000$ births, with only 6 to $25 \%$ of deletions having been inherited and the rest arising de novo (Wentzel et al., 2008; Grigorenko et al., 2010). Cat eye syndrome (arising from the supernumerary bisatellited marker chromosome) and $\operatorname{der}(22) \mathrm{t}(11 ; 22)$ syndrome are associated with either decreased or increased gene dosage (Edelmann et al., 1999a). 22q11.2DS is characterized by specific craniofacial features (cleft palate and velopharyngeal insufficiency, $69-100 \%$ ), thymic and parathyroid defects (17-60\%), mild to moderate renal anomalies (36-37\%), congenital cardiovascular malformations (49-83\%), and a range of cognitive and behavioral impairments (Grigorenko et al., 2010). Low average to borderline range of general cognitive functioning is one of the most consistent findings in the phenotypic presentation of 22q11.2DS (Antshel et al., 2008). Patients with duplications of the same region at 22q11.2 have been reported (de La Rochebrochard et al., 2006; Alberti et al., 2007; Yu et al., 2008). Although some of the features of the 22 q11.2 duplication syndrome overlap with the deletion syndrome, most of the symptoms are distinct and seem to vary compared to those of the 22q11.2 deletion syndrome (Wentzel et al., 2008).

Moreover, the whole phenotypic spectrum of 22q11.2 microduplication is not yet fully known, owing to a highly variable and mild phenotype, such that many individuals with microduplications may not undergo testing (Lo-Castro et al., 2009). The common molecular defect is a $\sim 3-\mathrm{Mb}$ deletion in $87 \%$ of the cases known as the "typically deleted region", followed by a proximally nested $\sim 1.5-\mathrm{Mb}$ deletion in $7 \%$, and other atypical deletions at chromosome 22q11.2 (Fernandez et al., 2009). Chromosome 22q11.2 is a well-characterized genomic region that is gene-rich and contains multiple region-specific low-copy repeats (LCRs) (McDermid and Morrow, 2002). Eight different LCRs are located in the proximal 22q region, which are presumed to predispose to mediate non-allelic homologous recombinations that result in interstitial deletions, duplications and inversions, as well as unbalanced translocations in this region (McDermid and Morrow, 2002). In theory, although both deletion and duplication events should occur in equal proportions, microduplications of the 22q11.2 region are about half as frequent as the deletions (Yu et al., 2008). Here we present and discuss the clinical and molecular findings of a 12-year-old girl with 46,XX karyotype and a 3.172-Mb duplication on chromosome 22q11.2.

\section{CLINICAL REPORT}

The propositus is a 12-year-old girl referred to the medical genetics department be- 
cause of mild mental-motor retardation and facial dysmorphism. She is the second child of a healthy 26-year-old mother and 32-year-old unrelated father. The first child of the family is healthy, and further family history was unremarkable. She was born by vaginal delivery at term; her birth weight was $3250 \mathrm{~g}$ (90th percentile), but length and head circumference were not recorded at that time. At 7 months old, stereotypic head movements were observed by her family. At 10 years old, her weight, length and occipitofrontal circumference were $25 \mathrm{~kg}$ (5-10th percentile), $127 \mathrm{~cm}$ (3rd-5th percentile), and $48 \mathrm{~cm}$, respectively, and at 12 years old, these were $37 \mathrm{~kg}$ (25-50th percentile), $145 \mathrm{~cm}$ (10-25th percentile), and $47 \mathrm{~cm}$, respectively. In her child history, eating difficulty, delayed walking without support at 5 years old, and speaking at 6 years old were pointed out. Although she was able to follow normal school without help, she was unsuccessful, and attention deficit disorder was noticed by her teachers. At present, she has hypernasal speech, delayed psychomotor development, and behavioral problems. Physical examination showed a mild facial dysmorphism consisting of a long narrow face, widely spaced eyes, downslanting palpebral fissures, broad nasal base, short philtrum, thin upper lip, micro/retrognathia, low set and retroverted ears, microcephaly, flat occiput, higharched palate, hypoplastic teeth, pectus excavatum, and bilateral hair loss on temporal regions of head (Figure 1). Assessment of her main organ systems, including cardiac, renal, urogenital, and endocrinological systems, was normal. Audiometric investigation of the patient was normal. Magnetic resonance images (MRI) of the brain showed vermian agenesis and ependymal cyst and mucosal thickness on maxillary sinus and ethmoid cellules.
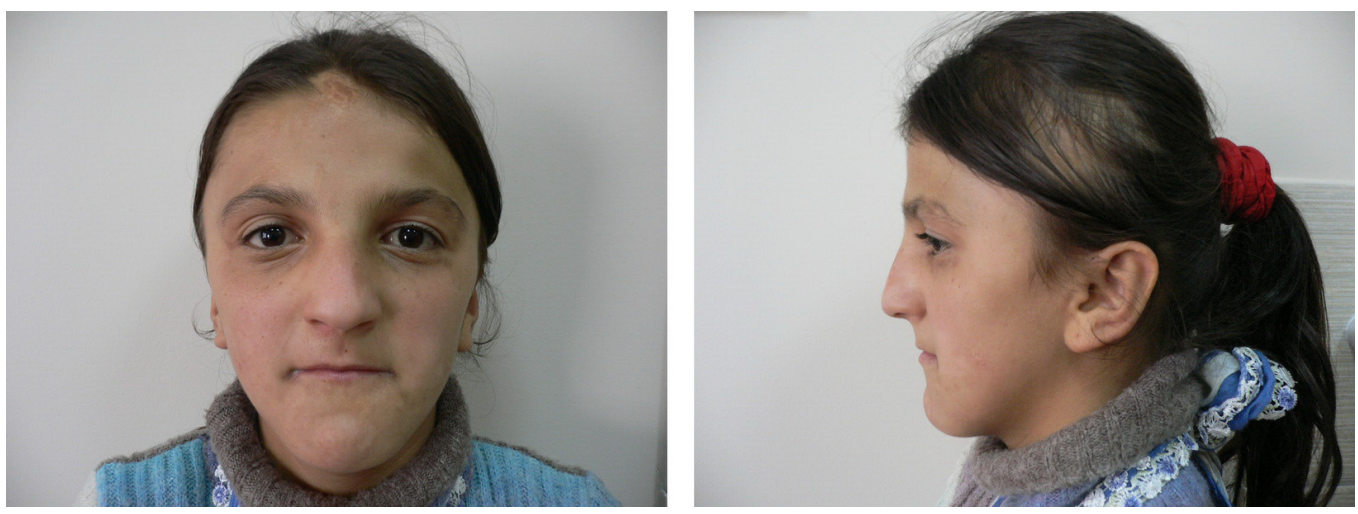

Figure 1. Image of the patient. Facial dysmorphism consisting of long narrow face, widely spaced eyes, downslanting palpebral fissures, broad nasal base, short philtrum, thin upper lip, and micro/retrognathia. Profile with low set and retroverted ears and hair loss on temporal region of her head is apparent.

\section{GENETIC ANALYSIS}

Metaphase slides of the patient and both parents were prepared from lymphocyte cultures of peripheral blood, and chromosome analysis was performed according to standard protocols using GTG banding at an approximately 550-band resolution. Standard chromosome preparations of the patient and her phenotypically healthy parents were analyzed, and the karyotypes were diagnosed as normal for all individuals. Since the karyotype analysis of 
the patient was normal, further investigation was performed by array-comparative genomic hybridization (array-CGH) to delineate any chromosomal anomaly. Parents' specimens were unavailable for array-CGH testing at the time of submission. A 3.172-Mb duplication at genomic position $17.041 .699 \rightarrow 20.213 .930$ bp in the 22 q11.2 region was detected by array$\mathrm{CGH}$, which was performed using a genome wide $180 \mathrm{k}$ oligo array platform (OGT CytoSure, OGT Oxford, UK) (Figure 2).

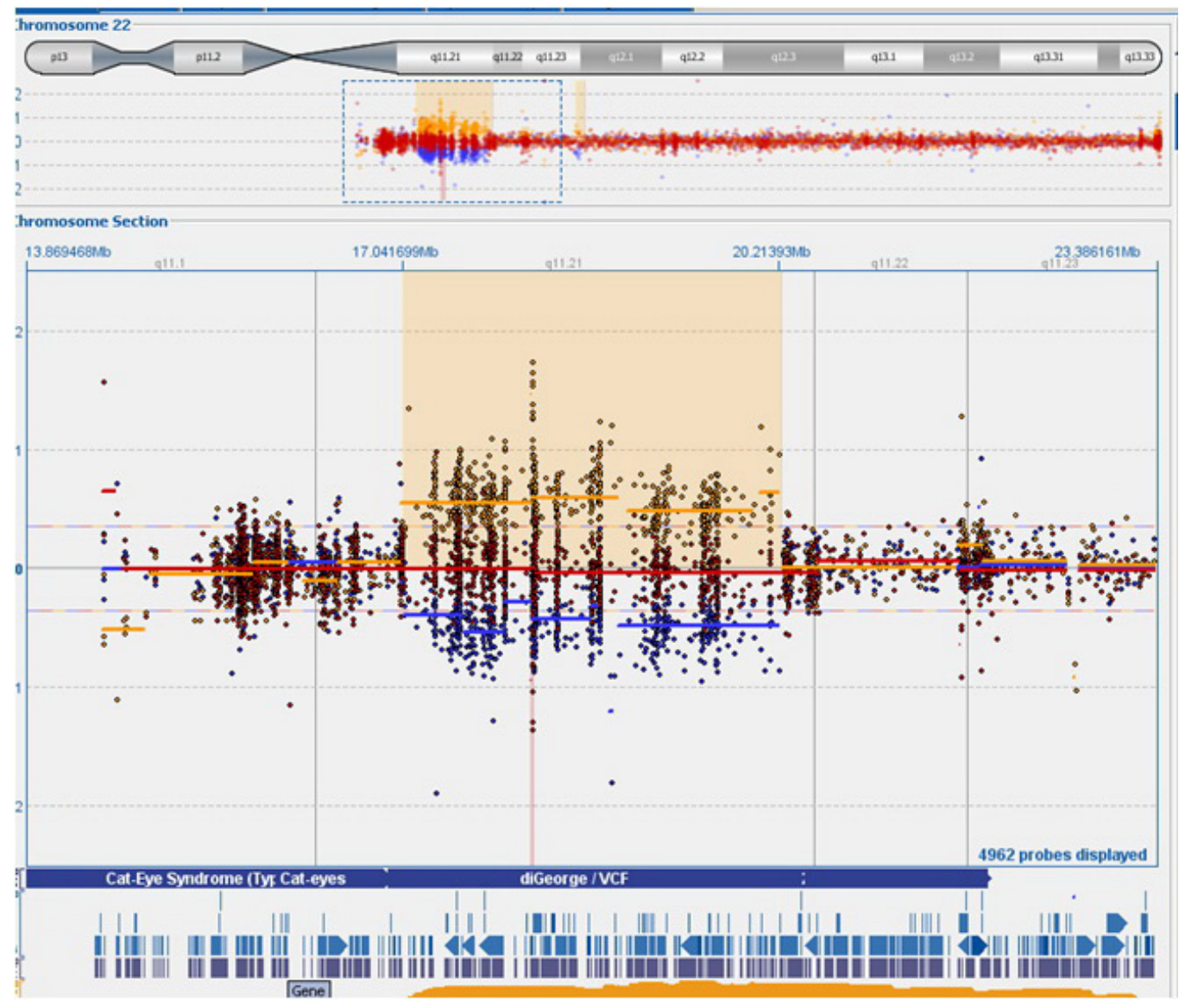

Figure 2. Oligoarray comparative genomic hybridization (CGH) study. Array-CGH showing a 3.172-Mb duplication in the 22q11.2 chromosomal region. $\mathrm{VCF}=$ velocardiofacial syndrome.

\section{DISCUSSION}

The 22q11.2 deletion syndrome's molecular mechanism has been well studied on the cytogenetic level; the deleted material can range from $3-\mathrm{Mb}$ deletion $(\sim 90 \%)$ to $1.5-\mathrm{Mb}$ deletion $(7 \%)$, with the rest having a smaller deletion. The $3-\mathrm{Mb}$ deletion region and the $1.5-\mathrm{Mb}$ region contain more than 45 and 24 genes, respectively (Grigorenko et al., 2010). The peri- 
centromeric region $22 \mathrm{q} 11$, is rich in regions of segmental duplication. In the $22 \mathrm{q} 11$ deletion syndrome, the typical 3-Mb deletion has both of its breakpoints in prominent segmental duplication regions (Grigorenko et al., 2010). The genes $P R O D H$ (the gene coding for proline dehydrogenase), TBX1 (member of the Tbox family of transcription factors), GNB1L (a gene that encodes a protein of unknown function), COMT (the gene encoding catechol-O-methyl transferase), $A R V C F$ (the armadillo repeat gene deleted in 22q11.2DS, which may have a role in cell-to-cell communication and intracellular transduction during embryonic development), $D G C R 8, R A N B P 1, Z D H H C 8$ (the gene coding for zinc finger- and DHHC domain-containing protein 8 ), and $P I K 4 C A$ are located in or close to regions of segmental duplication. There is a hypothesis that the syndrome is caused by an alteration of the genetic dosage of a particular gene or a number of genes in the region or distruption of a major regulatory gene, for example, the transcription factor TBX1 gene (Grigorenko et al., 2010; Scambler, 2010).

The phenotypes previously associated with microduplications of 22q11.2 are extremely variable with features overlapping those of the 22q11.2 deletion syndrome, including heart defects, velopharyngeal insufficiency with or without cleft palate, hypernasal speech, hearing loss, urogenital abnormalities, delayed growth and development, learning disabilities, behavioral problems, and various dysmorphic features (Portnoi, 2009). The mild facial dysmorphic features reported are hypertelorism, superior placement of eyebrows, epicanthal folds, broad nasal bridge, high forehead, downslanting palpebral fissures, smooth philtrum, midface hypoplasia, mild micro/retrognathia, dysplastic large ears, microcephaly, long and narrow facial shape, which were different from dysmorphic features of patients with DGS/ VCFC. In addition, a significant number of 22q11.2 microduplications may also be associated with a normal or near normal phenotype (Ensenauer et al., 2003). The reason for the wide phenotypic variation is unknown, but it has been speculated that epigenetic factors as well as non-penetrance may be involved (Yobb et al., 2005). In the recent years, several candidate genes reproducing the phenotype in animal models have been identified in 22q11.2 (Fernandez et al., 2009).

Until more information is available, perhaps we should consider the 22q11.2 syndrome as comprising only those individuals with common $3-$ or $1.5-\mathrm{Mb}$ proximally nested microduplications (Ou et al., 2008). The typical duplicated region of 3-Mb from LCR-A (LCR22-2) to LCR-D (LCR22-4) contains more than 40 genes, whereas the 1.5-Mb duplicated region extending from LCR-A (LCR22-2) to LCR-B (LCR22-3a) contains the TBX1 gene, which is a member of the T-box family of transcription factors; it has been shown to be the major disease gene in this region responsible for the DGS/VCFS when over- and under-expressed, explaining why there are many similar features between the 22q11.2 deletion and microduplication syndromes (Portnoi, 2009).

At least 63 individuals from 35 families have been described with duplication 22q11.2 (Courtens et al., 2008). In contrast to the reciprocal microdeletions (22q11.2 microdeletion syndrome), in which $90 \%$ occur de novo, familial transmission was reported in $70 \%$ by Wentzel et al. (2008). The first patient carrying a 3-Mb microduplication on 22q11.2 was a 4-year-old girl who presented moderate developmental delay, velopharyngeal insufficiency and a significant delay in language skills. Her facial features common to those of our patient were narrow face, downslanting palpebral fissures and velopharyngeal insufficiency (Edelmann et al., 1999b). Ensenauer et al. (2003) identified 10 unrelated patients with 22q11.2 microduplications, defining a new 22q11.2 microduplication syndrome distinct from the 22q11.2 deletion syndrome in 
a series of 653 patients. Ou et al. (2008) reported 19 individuals from 10 families with various sizes of genomic gains within the 22q11.2 region detected by array-CGH among 7000 samples referred for a variety of indications to undergo clinical testing over the course of several years. Among them, 10 individuals were found to carry 3-Mb microduplications of the common DGS/ VCFS region. The common clinical findings of these individuals, similar to those of our patient, were isolated speech delay, hypernasal speech and behavioral abnormality.

Common dysmorphic phenotypes similar to those of our patient were microcephaly, brachycephaly, downslanting eyes, micrognathia, and unusual hair patterns. In patients for whom parental samples were available, they observed a high frequency of familial transmission. Coppinger et al. (2009) screened 22,096 consecutive individuals with idiopathic mental retardation and/or congenital anomalies and reported 18 individuals with duplications of 22q11.21-q11.23. Thirteen of them were the first reported ones with 22q11.2 duplications that do not involve LCR22s A-D. Clinical records for nine subjects revealed shared characteristics, but some clinical features such as macrocephaly and upslanting palpebral fissures were contradicting clinical features (Coppinger et al., 2009). Lo-Castro et al. (2009) reported a 5.3-year-old girl with severe psychomotor retardation, facial dysmorphism, and autism, carrying a 22q11.2 duplication inherited from her mother who had a cleft palate and normal intelligence. She had severe mental retardation, severe autistic disorder and speech delay. Our patient has speech delay, learning disability, cognitive and behavioral disorders such as stereotypic movements of her head, anxiety, aggressivity, and attention deficiency, which are some of the major features of the 22q11.2 duplication syndrome. Courtens et al. (2008) reported on two families with 22q11.2 microduplication and, differently, they had thyroid problems, where both were treated with thyroid hormone. Our patient's thyroid hormone level was normal, but her mother had a thyroid operation and was treated with thyroid hormone. The occurrence of thyroid problem in the mother could be a coincidence, but the possibility of relationship with microduplication is not excluded. Prenatal testing is technically feasible but it is not possible to reliably predict the phenotype from a laboratory finding of 22q11.2 duplication (Wentzel et al., 2008).

The dysmorphic features of the patient presented here are likely the effect of $22 \mathrm{q} 11.2$ microduplication. Microduplication 22q11.2 may be largely undetected as a result of a nonspecific/mild phenotype and could be present more frequently in persons without clinical symptoms having a normal to even high intelligence (Courtens et al., 2008). Because it is undetectable by routine G-banded karyotyping, most individuals with 22q11.2 duplication are identified either by array-CGH testing, interphase FISH or multiplex ligation-dependent probe amplification testing for the 22q11.2 deletion syndrome (Stachon et al., 2007). Resolution of just a few hundred base pairs and even down to the single base pair level, as contrasted to conventional cytogenetic analyses with a resolution not better than several tens of thousands of base pairs, now allows high-resolution analyses of cryptic rearrangements (Grigorenko et al., 2010).

The molecular characterization of patients differs greatly between reports and detailed molecular characterization and documentation in the literature, making accurate genotypephenotype correlations difficult. Future reports of other families are needed to find out whether the delineation of this microduplication $22 \mathrm{q} 11.2$ is a true "syndrome" with a very large clinical variability and reduced penetrance or a non-pathogenic polymorphism (Courtens et al., 2008). In conclusion, our patient report will contribute to the growing knowledge regarding constantly new deletions and duplications of 22q11.2 region. 


\section{ACKNOWLEDGMENTS}

The authors would like to thank Prof. Dr. Sevim Balcı for helpful discussion and also the family of the patient who was the subject of this case study for the collaboration.

\section{REFERENCES}

Alberti A, Romano C, Falco M, Cali F, et al. (2007). 1.5 Mb de novo 22q11.21 microduplication in a patient with cognitive deficits and dysmorphic facial features. Clin. Genet. 71: 177-182.

Antshel KM, Fremont W and Kates WR (2008). The neurocognitive phenotype in velo-cardio-facial syndrome: a developmental perspective. Dev. Disabil. Res. Rev. 14: 43-51.

Coppinger J, McDonald-McGinn D, Zackai E, Shane K, et al. (2009). Identification of familial and de novo microduplications of 22q11.21-q11.23 distal to the 22q11.21 microdeletion syndrome region. Hum. Mol. Genet. 18: 1377-1383.

Courtens W, Schramme I and Laridon A (2008). Microduplication 22q11.2: a benign polymorphism or a syndrome with a very large clinical variability and reduced penetrance? - Report of two families. Am. J. Med. Genet. A. 146A: 758763.

de La Rochebrochard C, Joly-Helas G, Goldenberg A, Durand I, et al. (2006). The intrafamilial variability of the 22q11.2 microduplication encompasses a spectrum from minor cognitive deficits to severe congenital anomalies. Am. J. Med. Genet. A. 140: 1608-1613.

Edelmann L, Pandita RK, Spiteri E, Funke B, et al. (1999a). A common molecular basis for rearrangement disorders on chromosome 22q11. Hum. Mol. Genet. 8: 1157-1167.

Edelmann L, Pandita RK and Morrow BE (1999b). Low-copy repeats mediate the common 3-Mb deletion in patients with velo-cardio-facial syndrome. Am. J. Hum. Genet. 64: 1076-1086.

Ensenauer RE, Adeyinka A, Flynn HC, Michels VV, et al. (2003). Microduplication 22q11.2, an emerging syndrome: clinical, cytogenetic, and molecular analysis of thirteen patients. Am. J. Hum. Genet. 73: 1027-1040.

Fernandez L, Nevado J, Santos F, Heine-Suner D, et al. (2009). A deletion and a duplication in distal 22q11.2 deletion syndrome region. Clinical implications and review. BMC Med. Genet. 10: 48.

Grigorenko EL, Urban AE and Mencl E (2010). Behavior, brain, and genome in genomic disorders: finding the correspondences. J. Dev. Behav. Pediatr. 31: 602-609.

Lo-Castro A, Galasso C, Cerminara C, El-Malhany N, et al. (2009). Association of syndromic mental retardation and autism with 22q11.2 duplication. Neuropediatrics 40: 137-140.

McDermid HE and Morrow BE (2002). Genomic disorders on 22q11. Am. J. Hum. Genet. 70: 1077-1088.

Ou Z, Berg JS, Yonath H, Enciso VB, et al. (2008). Microduplications of 22q11.2 are frequently inherited and are associated with variable phenotypes. Genet. Med. 10: 267-277.

Portnoi MF (2009). Microduplication 22q11.2: a new chromosomal syndrome. Eur. J. Med. Genet. 52: 88-93.

Scambler PJ (2010). 22q11 deletion syndrome: a role for TBX1 in pharyngeal and cardiovascular development. Pediatr. Cardiol. 31: 378-390.

Stachon AC, Baskin B, Smith AC, Shugar A, et al. (2007). Molecular diagnosis of 22q11.2 deletion and duplication by multiplex ligation dependent probe amplification. Am. J. Med. Genet. A. 143A: 2924-2930.

Wentzel C, Fernstrom M, Ohrner Y, Anneren G, et al. (2008). Clinical variability of the 22q11.2 duplication syndrome. Eur. J. Med. Genet. 51: 501-510.

Yobb TM, Somerville MJ, Willatt L, Firth HV, et al. (2005). Microduplication and triplication of 22q11.2: a highly variable syndrome. Am. J. Hum. Genet. 76: 865-876.

Yu S, Cox K, Friend K, Smith S, et al. (2008). Familial 22q11.2 duplication: a three-generation family with a 3-Mb duplication and a familial 1.5-Mb duplication. Clin. Genet. 73: 160-164. 